PROBLEMS OF ENGINEERING CYBERNETICS AND ROBOTICS • $2021 \bullet$ Vol. 77, pp. 3-10

p-ISSN: 2738-7356; e-ISSN: 2738-7364

https://doi.org/10.7546/PECR.77.21.01

\title{
Estimation of Volatility based on the Estimation of Segmentation
}

\section{Todor Balabanov}

Institute of Information and Communication Technologies

Bulgarian Academy of Sciences

Acad. Georgi Bonchev Str., block 2, office 514

1113 Sofia,Bulgaria

todor.balabanov@iict.bas.bg

\begin{abstract}
The most common games of chance are slot machines where the virtual reels are rotated even when reels stop screen with prizes is shown. The arrangement of the symbols on the reels sets the discrete probability distribution of the game. One of the important characteristic of such games is the ratio between total loss and total bet that determines the return to player measured in percent. The other important characteristic of these games is its volatility. This indicator shows how much the game deviates from the average tendency. Due to volatility importance, in the current article an estimation based on approximation of the virtual reels with segments from the original ones is discussed.
\end{abstract}

Keywords: reverse engineering, slot machines, volatility index.

\section{Introduction}

In the gambling industry, the most popular games are slot machines or also known as one-armed bandits. When they were originally invented, slot machines consisted of mechanical, rotating reels. The player pulled a lever that activated the spin of the reels. When stopped, the reels visualized a line of drawn symbols in front of the player. Each of the symbols is characterized by the probability of occurrence and the amount of profit it brings. With the rapid development of computer technology, slot machines from electro-mechanical gradually became electronic or computerized. 
Computerized slot machines retain the principle of reels with pre-arranged symbols. Reels are virtual because they are represented in the machine in the form of numbers in electronic memory. The exact arrangement of the symbols is determined by a game designer in close collaboration with a mathematician (Petrov et al., 2020a). The arrangement itself is a discrete probability distribution (Petrov et al., 2020b). Symbols paying lower profits are more common, and symbols paying higher profits are significantly less common. The exact value of the winning combinations is set in advance in a game paytable.

Modern slot machines show several rows from the virtual reels. The most common games have three, five, or more reels. Winnings are formed from predefined lines or winning patterns (Tomov et al., 2017). In this way, not only the centreline, the one above it, and the one below it is profitable, but diagonals, irregular lines, zigzag combinations, and others are possible.

In computerized slot machines, in addition to the base game (Keremedchiev et al., 2017), there are often free spins and various bonus games. The ratio of the total amount won to the total amount bet, multiplied by one hundred (Kamanas et al., 2021), determines the characteristic return to the player (RTP) in percentage. The RTP indicator has the meaning of mathematical expectation (Balabanov et al., 2015b). RTP determines how many of the 100 bets would return, on average, for one spin of the reels (Balabanov et al., 2015a). For example, in a game that is characterized by $98 \%$ RTP, betting 100 coins leads to a mathematical expectation that 98 of them will return as a win. Slot machines are mathematically unfair games, as the player's chances are always less than the gaming hall. Various authorities around the world allow RTP values to range from $75 \%$ to $98 \%$. It is economically unjustifiable for gambling operators to exceed $100 \%$ of RTP. With RTP above 100, the hall would lose more than it would gain. In addition to the RTP feature, each slot machine is also characterized by volatility. While RTP is a parameter strictly monitored by legal regulators, volatility is not always required and monitored. The volatility of the game determines how often the winnings fall and what their size is. Slot machines are often manufactured according to the social attitudes in the society in which the game will be distributed and played. For example, American players are significantly more risky than players in other countries. They prefer more highly volatile games. In Eastern Europe, there are preferences for less volatile games. High-volatility games yield significantly less often, but falling profits are of great value. Low-volatility games give profits significantly more often, but these winnings are smaller. The volatility of the game is often marked with one to five stars, from low to high.

There exist variety of approaches capable to cope with different kind of uncertainty. Some of them rely on well-known optimization strategies proposed by Wald, Laplace, Hurwitz, and Savage and their modifications (Borissova \& Dimitrova, 2021) or cost-benefit analysis (Borissova et al., 2017). In some cases, it is possible to use the heuristic approaches (Guliashki, 2006). Review of the 
uncertainty measures in evidence theory with an analysis of some related controversies can be found in (Deng, 2020). In situations where prior information is available on parameters, the uncertainty can be measure considering this prior information as shown in (Patra, 2020).

The present study proposes a way to empirically determine volatility without knowing the complete and exact location of the symbols on the reels. With such a level of uncertainty, some effort is needed to collect the necessary statistical information. After the introduction, the article is organized as follows: Section 2 explains the idea of statistical estimation through reels' segments; Section 3 presents the experiments performed and the results obtained; Section 4 concludes and provides guidelines for future development.

\section{Estimation by Segments}

The volatility of a slot machine is of interest when the gambling product is inspected by regulators or tested by competing manufacturers. In the legal regulation of slot machines, manufacturers provide complete and detailed documentation for each product. This documentation also includes the exact values on the virtual reels. Given the paytable, the virtual reels, the winning combinations, and the rules for free spins and/or bonus games, it is a matter of combinatorial calculation to calculate RTP and volatility. However, when the manufacturer's documentation is not available, only the pay-out table, pay lines/patterns, free spins rules, and bonus game rules are known. When virtual reels are not known (Tomov et al., 2017), this requires some form of empirical research to determine RTP and volatility.

A rough estimate of the value of RTP can be made by repeatedly starting the spins of the reels. Some research laboratories (for example GLI), roughly determine the value of RTP by playing 14K spins taking into account the level of total win, the level of the total bet, and calculating the ratio between the two. Volatility as a kind of estimate of deviation from the central tendency can be calculated by following relation:

$$
\mathrm{V}=\mathrm{C} \sqrt{\frac{\sum H_{i}\left(P_{i}-R\right)^{2}}{N}}
$$

where $V$ is the volatility index, $C$ is the confidence interval, $H_{i}$ is the frequency of the $i$-th winning combination, $P_{i}$ is the value of the $i$-th win, $R$ is the value of RTP, and $N$ is the number of spins. It is important to note that volatility is calculated by playing only one line (usually the central one). Most of the modern slot machines are rarely played in a single-line configuration. Single-line configuration is not even possible in most games.

In the most common game screen configuration, slot machines have 5 reels and 3 rows. The presence of 15 symbols $(5 \times 3)$ visible on the screen allows the 
formation of many different patterns for pay-out. When the exact virtual reels are known, enough statistics can be collected by Monte Carlo simulation to estimate the volatility. When virtual reels are unknown, information can be gathered through many observations of different spins/stops and to be used. Stopping the virtual reels reveals authentic snippets of the order of the symbols. Most often, the animation visualizing the illusion of rotating reels does not contain real information about the location of the symbols but contains images that create the best possible illusion of rotation. For this reason, the only information that can be considered reliable and authentic is the state of the game screen after stopping the spin. It is also important to note that games must be explored in real mode, with real bets, as demonstration modes are often performed with a different set of virtual reels.

Monte Carlo simulations of slot machines are performed by stopping the virtual reels at specific random positions. The previous symbol and the following symbol relative to the random stop are also taken (Balabanov, 2021). In the absence of complete information about the virtual reels, the observation of the segments obtained at different stops of the reels is proposed. The resulting segments give a statistical idea of the actual location of the symbols. In the simulation, the playing screen is not formed by the reels, but segments are selected based on the frequency of their encounter (Balabanov, 2021).

\section{Experiments and Results}

In order to conduct successful experiments, it is essential that the calculated volatility be compared between the actual reels of the slot game and the reels approximated with segments. For this purpose, a publicly presented and publicly analyzed slot machine is selected (Dimov, 2012). Bug A Boom is a demo slot game that has been analyzed as RTP and volatility. The game has five reels and three rows visible on the screen. Winnings are formed by twenty lines, which are accounted from left to right. When there is more than one win on a line, only the biggest win is taken. The game also has a scatter symbol that brings wins anywhere on the screen. Scatter has no need to participate in a specific pay line. Fifteen free spins are triggered on three scatters, which bring winnings multiplied by four. Additional free spins may occur during the free spins themselves. Different sets of virtual reels are used for the base game and for the free spins. There is also a wild symbol in the game, which replaces any other symbol in a winning combination except the scatter symbol. When the wild is used as a substitute, the winnings are multiplied by two. If the winning combination is formed only by wilds, the winnings are not doubled. Wild symbols and scatter symbols cannot appear simultaneously in the same spin/stop. The RTP percentage was experimentally calculated from the creator of the game model. It was estimated as $94.77 \%$. Knowledge of the RTP set by the manufacturer allows the proposed way for approximation with segments to be effectively applied, 
observing the statistical sample of the segments to lead to a similar value of RTP. The volatility of the game was determined by its creator as high (4 out of 5). This fact is also confirmed by the paytable, where higher values of winnings formed by five symbols are clearly observed, compared to the lower values of winnings formed by four symbols. For 20 betting lines, the total bet per spin is 20 coins. It is characteristic of highly volatile games is that the smallest winnings are below the value of the total bet. In this game, the first seven symbols participating in the triple-winning combination, and the remaining five symbols participating in the double-winning combination, have winnings of 2 to 15 coins, which is under the total bet.

Although the description of the mathematical model is publicly available, the model alone cannot estimate the mean (RTP) and the deviation from the mean (volatility). To make these estimates, program code has been developed. It integrates the game model and performs Monte Carlo simulation for statistics collection (Balabanov, 2021). The simulator allows anyone to study the RTP and the volatility at different numbers of spins. All experiments in the current study are performed at $100 \mathrm{M}$ spins.

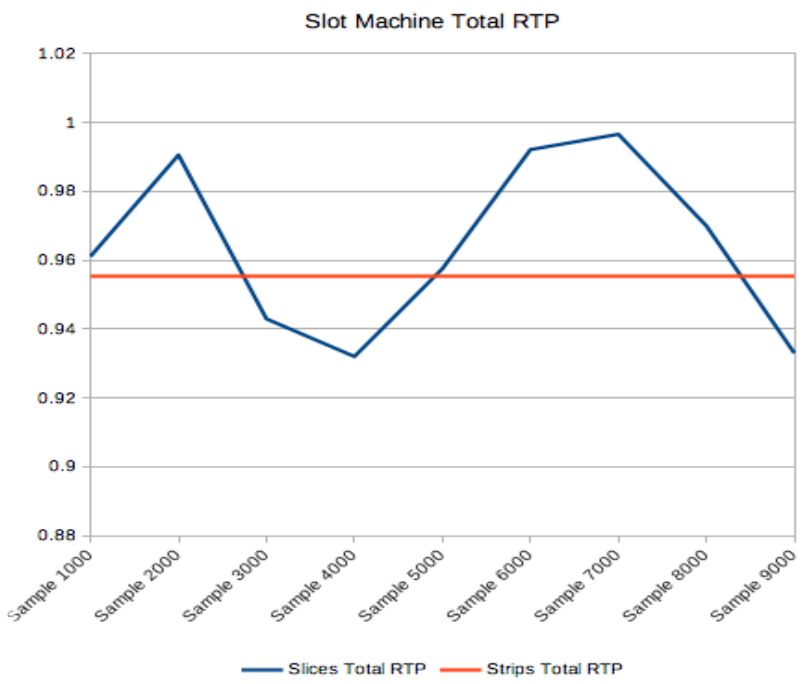

Fig. 1. Total RTP of the game

From the graph of Fig. 1, it is clear that the simulation with the original reels approaches the RTP announced by the manufacturer. When approximating the reels with segments, statistical samples with sizes from 1000 to 9000 observations are used. Inevitably, the use of segments introduces additional inaccuracies in determining the RTP percentage. In industrial development, it is important to calculate volatility with a sample of segments, which leads to very similar results in terms of the already known RTP percentage. It is important to note that the larger the statistical sample is, the more accurate the empirical determination of 
RTP and volatility would be. In real practice, generating a large sample involves a large amount of work and often proves impractical.

Volatility is calculated when only one pay line is active. This means that the other 19 lines are deactivated artificially. As shown in Fig. 2, the volatility calculated with the original reels goes close to the approximate ones, but it has variations.

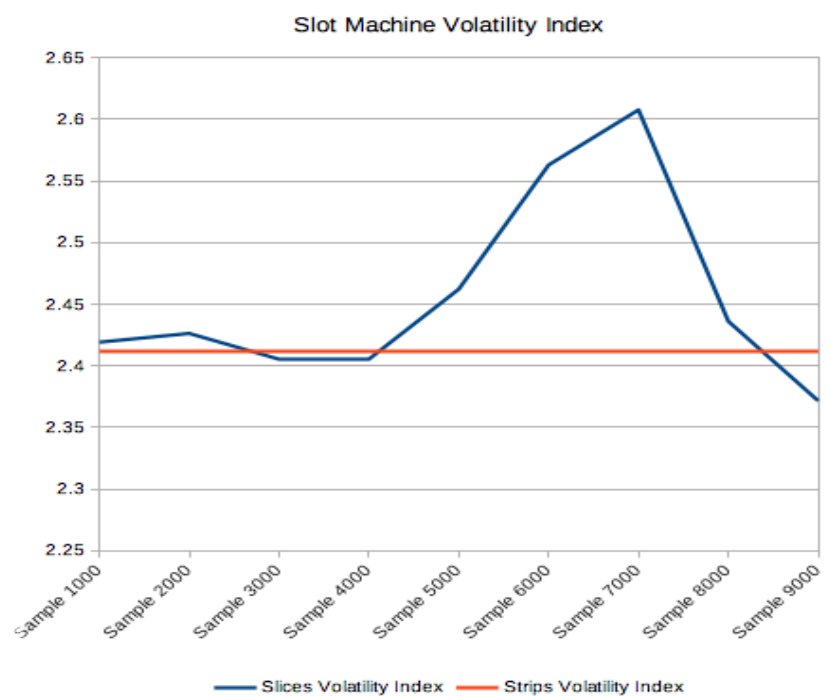

Fig. 2. Base game volatility

Volatility is calculated only with respect to the virtual reels in the base game, which is illustrated by the corresponding partial RTP in Fig. 3.

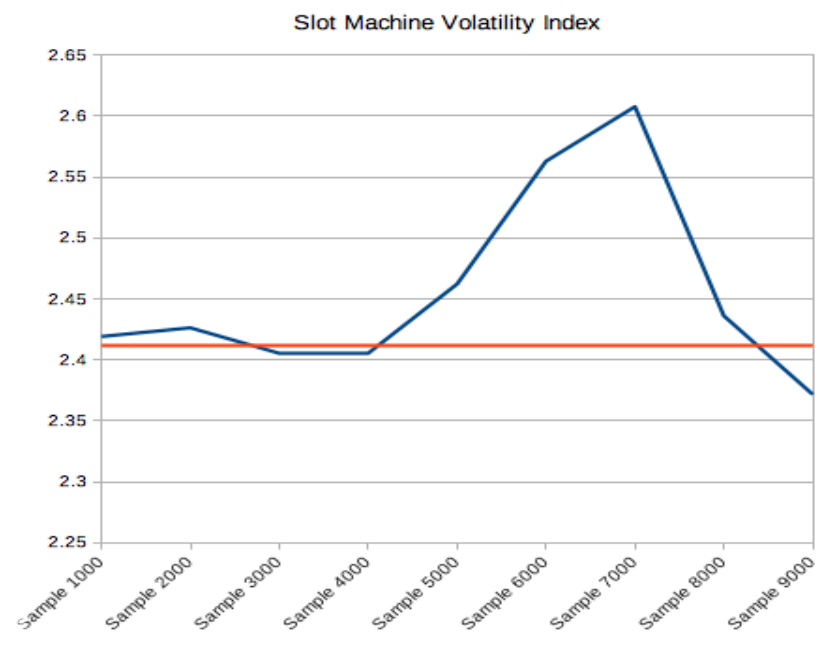

Fig. 3. Base game RTP for a single line configuration 
In this graph, it is clear that the approximation of the virtual reels leads to deviations in values from those calculated with the original reels. Finding the optimal value for the size of the statistical sample is a matter of repeated experiments. The leading criteria for determining the sample size is reaching a close value for the total RTP, compared to that announced by the manufacturer.

\section{Conclusion}

In the present study, a way for estimation of the mathematical expectation and its deviation is proposed. The approximate calculation is achieved by observing segments of the original virtual reels. The experiments performed clearly show that the proposition could find real industrial application in gambling slot machines analysis.

The main advantage of the proposal is that it automates work usually done manually. When done by hand, it takes an unacceptably long time which makes it industrial inapplicable. The main disadvantage of the proposal is that combinatorial optimization is extremely difficult when the aim is to reach the global minimum for the length of the reels. The results of the optimization are very dependent on the sample size.

The need for automated recording of the observed segments can be pointed out as guidelines for future development. This type of automation could be achieved by tools of computer graphics and image recognition.

\section{Acknowledgments}

This research is funded by Velbazhd Software LLC and it is partially supported by the Bulgarian Ministry of Education and Science (contract D01205/23.11.2018) under the National Scientific Program „Information and Communication Technologies for a Single Digital Market in Science, Education and Security (ICTinSES)“, approved by DCM \# 577/17.08.2018.

\section{References}

1. Balabanov, T., Zankinski, I., Shumanov, B.: Slot machine RTP optimization and symbols wins equalization with discrete differential evolution. In: Proc. of International Conference on Large-Scale Scientific Computing, vol. 9374, pp. 210-217 (2015). https://doi.org/10.1007/978-3-319-26520-9_22.

2. Balabanov, T., Zankinski, I., Shumanov, B.: Slot machines RTP optimization with genetic algorithms. In: Proc. of 8th International Conference Numerical Methods and Applications, vol. 8962, pp. 55-61 (2015). https://doi.org/10.1007/978-3319-15585-2_6.

3. Balabanov, T.: Approximated 20 burning hot slot game simulation version 1.0.0. ResearchGate (2021). DOI https://doi.org/10.13140/RG.2.2.18878.00329.

4. Balabanov, T.: Bug A Boom Simulator. Velbazhd Software LLC (2021). http://github.com/VelbazhdSoftwareLLC/BugABoomSimulator. 
5. Balabanov, T.: Fruit machine simulator with Excel input-output interface. Velbazhd Software LLC (2021), https://github.com/VelbazhdSoftwareLLC/Fruit-Machine-Simulator-with-ExcelInterface/blob/c7c48b6fad30e2554356ae04dc70f9e54cc99984/src/main/java/eu/ veldsoft/slot/simulator/Simulation.java\#L1125.

6. Borissova, D., Dimitrova, Z.: An integrated group decision-making approach considering uncertainty conditions. In: Proc. of 24th International Conference on Business Information Systems (BIS'2021) pp. 307-316 (2021), https://doi.org/10.52825/bis.v1i.52.

7. Borissova, D., Mustakerov, I., Korsemov, D., Dimitrova, V.: Selection of ERP via cost-benefit analysis under uncertainty conditions. Advanced Modeling and Optimization 19(2), 177-186 (2017).

8. Deng, Y.: Uncertainty measure in evidence theory. Science China Information Sciences 63, 210201 (2020). https://doi.org/10.1007/s11432-020-3006-9.

9. Dimov, V.: Bug A Boom. SLOT Design Consult Ltd. (2012). https://www.slotdesign.com/simulator/Bug A Boom Desc.pdf

10. Guliashki, V.: The two-group classification problem challenge - Polynomial Heuristic Algorithms. Cybernetics and Information Technologies 6(2), 43-53 (2006).

11. Kamanas, P., Sifaleras, A., Samaras, N.: Slot machine RTP optimization using variable neighborhood search. Mathematical Problems in Engineering, 8784065 (2021). https://doi.org/10.1155/2021/8784065.

12. Keremedchiev, D., Tomov, P., Barova, M.: Slot machine base game evolutionary RTP optimization. In: Proc. of International Conference on Numerical Analysis and Its Applications, vol. 10187, 406-413 (2017). https://doi.org/10.1007/978-3319-57099-0 45.

13. Patra, L. K., Kayal, S., Kumar, S.: Measuring uncertainty under prior information. IEEE Transactions on Information Theory 66(4), 2570-2580 (2020), https://doi.org/10.1109/TIT.2020.2970408.

14. Petrov, P., Kostadinov, G., Zhivkov, P., Velichkova, V., Balabanov, T.: Approximated sequences reconstruction with genetic algorithms. In: Proc. of 28th International Symposium - Management of Energy, Industrial and Ecological Systems, 63-66 (2020).

15. Petrov, P., Kostadinov, G., Zhivkov, P., Velichkova, V., Balabanov, T.: Approximate sequencing of virtual reels with genetic algorithms. In: Proc. of 23rd International Conference on Distributed Computer and Communication Networks: Control, Computation, Communications, 507-514 (2020).

16. Tomov, P., Zankinski, I., Balabanov, T: Slot machine reels reconstruction with Monte-Carlo search. In: Proc. of International Scientific Conference UniTech, vol. 2, pp. 384-387 (2017).

17. Tomov, P., Zankinski, I., Balabanov, T: Slot machine reels reconstruction with Genetic Algorithms. Extended abstracts of Annual Meeting of the Bulgarian Section of SIAM, 102-103 (2017). 